IdeAs

Idées d'Amériques

$2 \mid 2012$

Universités d'Amériques et construction des savoirs

\title{
Regards sur un campus nord-américain, UCLA
}

\section{Capucine Boidin et Cynthia Ghorra-Gobin}

\section{OpenEdition}

Journals

Édition électronique

URL : https://journals.openedition.org/ideas/344

DOI : 10.4000/ideas.344

ISSN : 1950-5701

\section{Éditeur}

Institut des Amériques

\section{Référence électronique}

Capucine Boidin et Cynthia Ghorra-Gobin, « Regards sur un campus nord-américain, UCLA », IdeAs [En ligne], 2 | 2012, mis en ligne le 22 juin 2012, consulté le 19 octobre 2022. URL : http:// journals.openedition.org/ideas/344 ; DOI : https://doi.org/10.4000/ideas.344

Ce document a été généré automatiquement le 19 octobre 2022

\section{(c) (i) (9)}

Creative Commons - Attribution - Pas d'Utilisation Commerciale - Pas de Modification 4.0 International - CC BY-NC-ND 4.0

https://creativecommons.org/licenses/by-nc-nd/4.0/ 


\title{
Regards sur un campus nord- américain, UCLA
}

\author{
Capucine Boidin et Cynthia Ghorra-Gobin
}

\begin{abstract}
La réputation des universités nord-américaines est universellement reconnue. Comme il s'agit d'un pays fonctionnant pratiquement à l'échelle continentale, sur quels critères se joue la diversité ? N'y observe-t-on pas au-delà de la diversité un modèle de campus américain?

Cynthia Ghorra Gobin: Il est plus simple d'évoquer dans un premier temps la diversité des universités avant de parler du campus américain en tant que modèle. Le campus varie en fonction de la taille de l'université, de la date de création du campus, de la localisation du campus par rapport à la ville (dans la ville ? à l'extérieur ? en périphérie?), de la spécialisation académique (université centrée sur les undergraduate students? sur les graduate students? ou sur les deux ?) et du classement (ranking) de l'université dans le système national. L'université est-elle réputée pour sa business school? son centre de recherches sur la gestion des écosystèmes naturels? la recherche bio-médicale ? Certains campus remontent à la période coloniale comme Harvard alors que d'autres comme Stanford ou UC Berkeley (toutes deux localisées dans l'aire urbaine de San Francisco) remontent à la fin du XIX ${ }^{\text {ème }}$ siècle. Le campus de l'Université de Californie à Los Angeles (UCLA) est localisé dans un quartier chic de la ville alors que celui de Columbia à New York se situe à l'interface d'un quartier aisé et de Harlem.
\end{abstract}




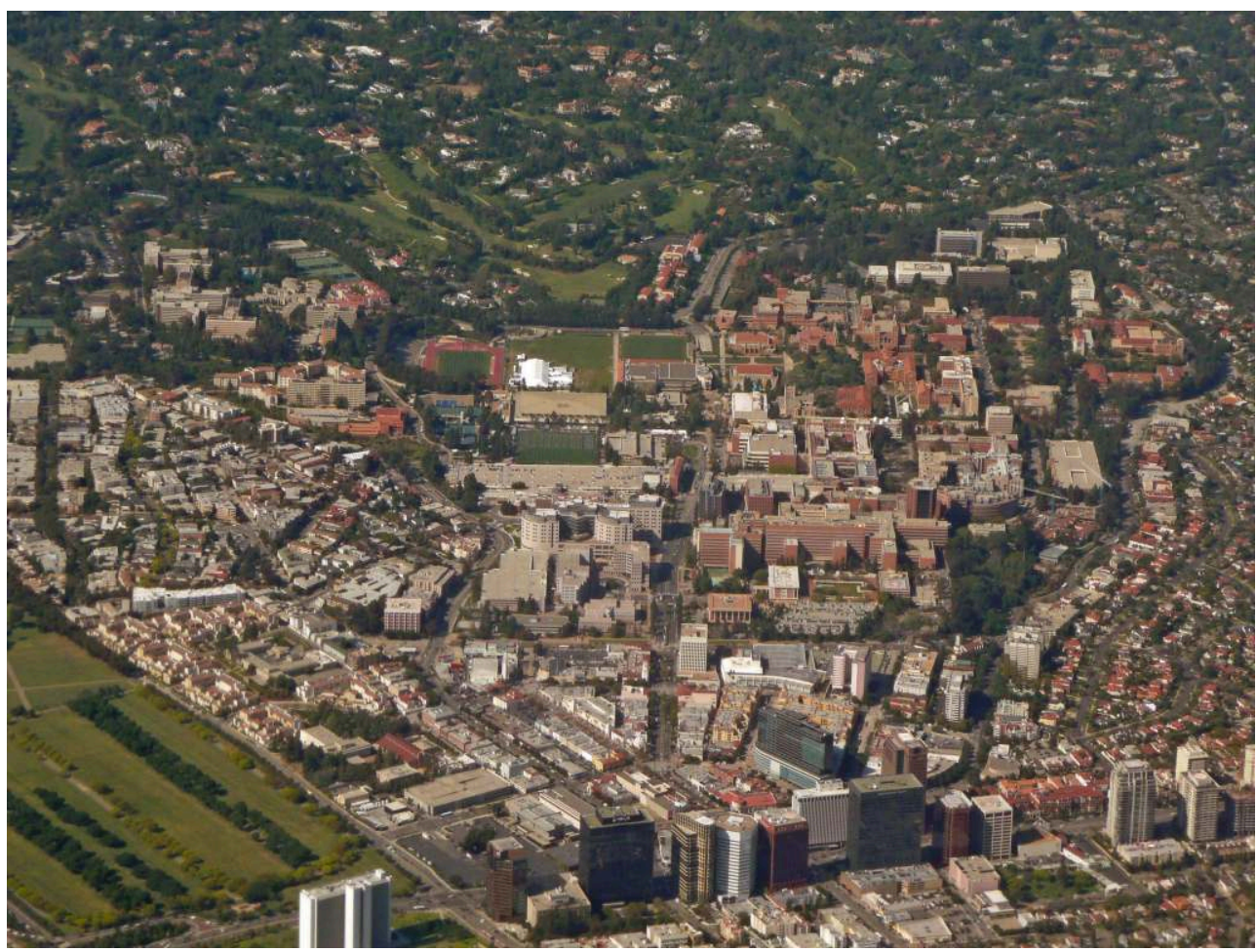

ATIS547

Il y a bien une grande diversité d'universités (publiques et privées) mais leur similitude se lit au niveau de l'organisation spatiale du campus, que ce dernier relève du secteur privé ou du secteur public. Dans la métropole de Los Angeles, les campus de CalTech (California Institute of Technology) -Université prestigieuse dont le chancelier est un French-American- et de l'Université de Californie du Sud (USC) ne sont pas tellement différents de celui de UCLA, alors que les deux premiers relèvent du secteur privé et la troisième du secteur public. Parmi les universités publiques, on distingue également la land-grant university ayant bénéficié d'un terrain offert par l'État fédéré à l'université. La land-grant university se retrouve plus facilement dans les états du midwest parallèlement à la conquête territoriale. Le prix de toute scolarité est nettement plus élevé dans une université privée que dans une université publique mais toutes les deux attribuent des bourses aux étudiants qu'elles souhaitent recruter et qui en font la demande.

Reconnaître la diversité des universités américaines signifie aussi prendre conscience du classement dans lequel s'inscrivent ces universités, y compris au sein de chacune des disciplines. Aussi tout étudiant(e) -comme tout enseignant(e) connaît le positionnement de son département et de son université dans le palmarès des universités américaines. Cette connaissance lui permet d'avoir ainsi une idée relativement précise du marché de l'emploi. Si il ou elle décide de faire de la recherche, le choix des thématiques se fera sur la base de la réputation du département et en fonction d'un futur recrutement dans une autre université. Le système américain tolère peu qu'un étudiant ayant obtenu un Ph.D. dans une université donnée y soit embauché. Par exemple, à la fin des années 1970 et au début des années 1980, tout étudiant au département d'études urbaines savait que celui-ci 
se classait au $5^{\text {ème }}$ rang après ceux de Harvard, MIT, Cornell et UC Berkeley. Mais audelà de la hiérarchie et de la diversité universitaire, le dessin du campus américain présente quelques similarités.

Mais au fait quelle est la première impression que vous avez eue du campus de UCLA à votre arrivée à Los Angeles en 1978 ? Comment se repère-t-on ? Faut-il être piéton ou automobiliste?

Cynthia Ghorra Gobin : Le campus de UCLA est localisé à Westwood, un quartier chic de la ville de Los Angeles situé à l'ouest de la ville et pas trop éloigné de l'océan. Ce qui signifie que le smog y est moins sévère que dans le centre-ville et à South Central (ghetto noir), que les températures sont clémentes en raison de la brise venue de l'océan qui rafraîchit les températures en été et dissipe le smog. La première fois que je me suis rendue sur le campus fut en voiture en passant par Sunset Boulevard (une avenue qui traverse plusieurs quartiers de la ville) qui conduit aux deux entrées nord du campus. Vivre à Los Angeles exige de prendre le temps de se familiariser avec les circuits et horaires des bus. L'entrée principale se trouve au sud du campus à proximité du boulevard de Wilshire - l'axe central de la ville se déployant du centre ville situé plus à l'est jusqu'à l'océan à l'ouest.

Dès l'entrée du campus des panneaux indiquent la localisation du parking le plus proche, ce qui permet à l'automobiliste de ne pas perdre de temps et ainsi de devenir très rapidement piéton ${ }^{1 .}$ La marche à pied est sans aucun doute le meilleur moyen de se déplacer sur le campus, qui en raison de son étendue exige de se munir d'un plan indiquant la localisation des principaux bâtiments et des cheminements pour y accéder. A Los Angeles se retrouver sur un campus où tout le monde (ou presque tout le monde) circule à pied ou en vélo s'avère une expérience assez originale. Il est vrai que L.A.se présente comme une ville où la majorité des déplacements se font en voiture, en dépit d'une sérieuse expansion des transports en commun et du covoiturage au cours de ces vingt dernières années.

L'autre forte impression du campus réside sans aucun doute dans le caractère verdoyant. Les voies où circulent quelques voitures ainsi que les allées principales sont bordées d'arbres. La pelouse est omniprésente sur le terrain qui sépare les bâtiments sans oublier l'aire centrale, à proximité du grand auditorium et de la bibliothèque. La pelouse figure certes en tant qu'élément du décor ou du paysage mais elle représente également un espace que peuvent s'approprier les étudiants pour travailler, discuter ou encore partager un lunch (milieu de la journée) ou un snack (en cours de journée). Il est vrai qu'en Californie du Sud, les conditions climatiques permettent de profiter de la pelouse onze mois sur douze. Ce caractère verdoyant se retrouve à des degrés divers dans tous les campus américains.

La conception du campus relève ainsi d'un sérieux dosage d'urbanisme paysager et d'urbanisme architectural. En d'autres termes les arbres et la pelouse ne sont pas là pour remplir le vide entre les bâtiments mais représentent plutôt une structure dans laquelle s'insèrent les bâtiments. Cet agencement végétal-minéral qui caractérise tout campus américain est largement influencé par cette vision idéalisée de la nature remontant au $19^{\text {ème }}$ siècle, siècle au cours duquel le peuple américain se forge son identité. 
Cette impression est-elle toujours la même toutes les fois que vous vous rendez à Los Angeles? Pourquoi ?

Cynthia Ghorra Gobin: Cette première impression d'un cadre verdoyant et principalement fréquenté par les piétons est toujours valable quand je me rends à UCLA. Force est toutefois de constater qu'au cours des 15 dernières années le campus a subi un processus de densification en raison de la croissance des effectifs de recherche dans certains départements. En d'autres termes le nombre de bâtiments a augmenté notamment le long du boulevard urbain qui entoure le campus. Il a fallu répondre à la demande de centres de recherche et au nombre croissant d'étudiants et de professeurs, notamment dans le secteur de la biotechnologie. Le complexe hospitalier, qui comprend de nombreuses unités de recherches, concentre une grande partie des financements publics (Etat fédéral) et privés reçus par l'université. La densification du campus n'a pas pour autant fait disparaître le caractère verdoyant de l'ensemble puisque la majorité des nouveaux bâtiments se retrouvent en périphérie. Le campus continue d'appartenir en priorité au piéton. Certes l'offre de places de places parking (notamment pour ceux pratiquant le voiturage) s'est accrue mais elle ne se lit pas dans le paysage.

Le campus s'inscrit bien dans un cadre paysager et de ce fait confirme cette tradition américaine remontant à l'époque des philosophes transcendantalistes (Emerson et Thoreau) de la première partie du XIX ${ }^{\text {ème }}$ siècle qui ont insisté sur la présence de la nature comme possibilité de ressourcement pour l'individu et comme lieu privilégié de l'identité américaine. Ces fondements philosophiques ont conduit l'action de nombreux architectes et paysagistes comme Frederick Law Olmsted qui a réalisé Central Park à New York et le campus de Stanford (comté de Santa Clara au sud-est de la ville de San Francisco). Les premiers campus en Amérique ont été conçus à l'image des campus britanniques comme Harvard qui se veut une réplique d'Oxford ou de Cambridge. Mais au XIX ${ }^{\text {ème }}$ siècle, architectes, urbanistes et paysagistes conçoivent et réalisent des campus en favorisant l'élément paysager plutôt que l'architecture. Notons toutefois que le bâtiment qui inclut la bibliothèque se reconnaît à son architecture monumentale et imposante. Le campus de l'UCLA inclut deux grandes bibliothèques, une pour les étudiants non gradués et l'autre pour les étudiants gradués et les chercheurs. A ces deux bibliothèques s'ajoutent bien entendu des bibliothèques plus petites appartenant à des départements et de ce fait plus spécialisées et plus limitées. 


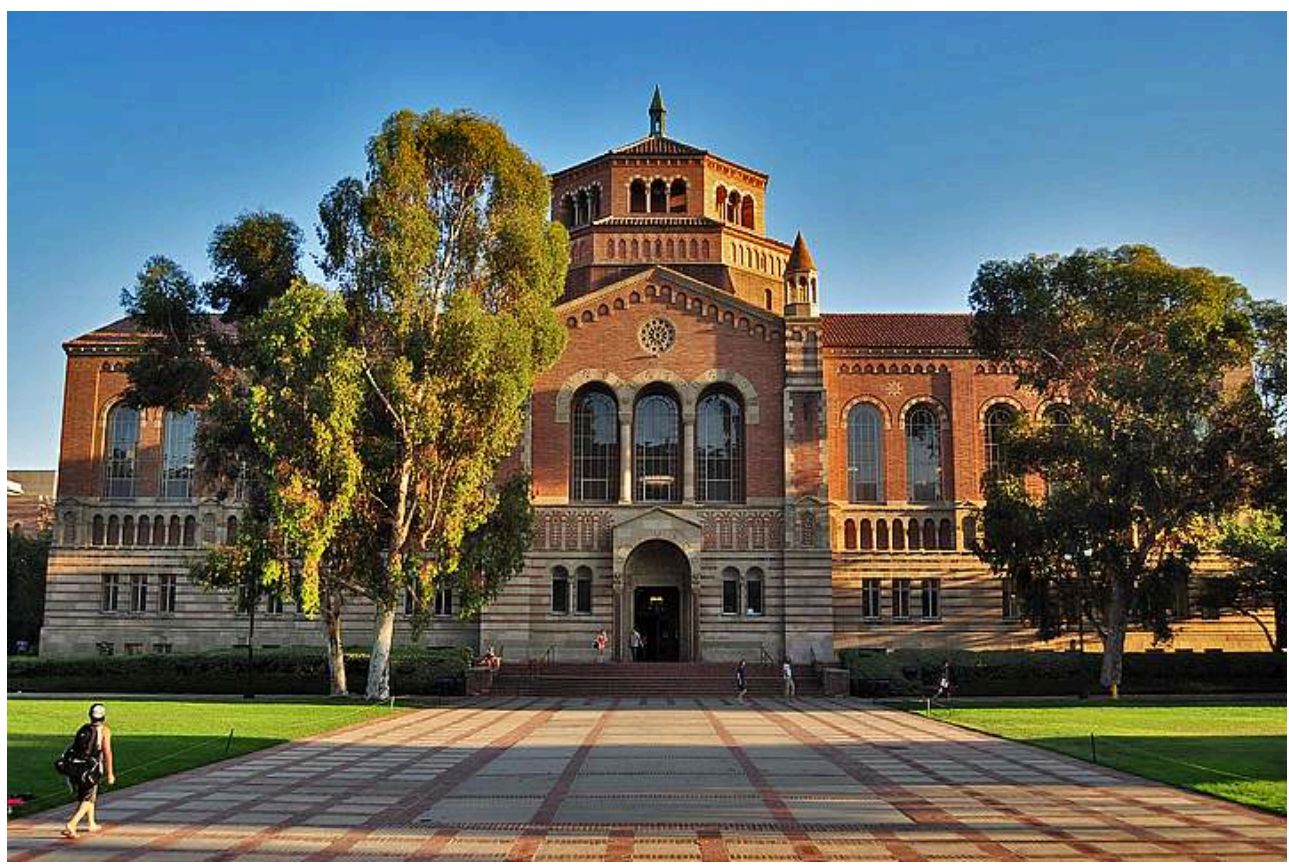

Campus Grotto

D'après ce que vous dites, le campus connaît une forte animation durant la journée et durant la semaine. Mais qu'en est-il le soir? le week-end? qui vit sur le campus ?

Cynthia Ghorra Gobin : Le campus donne l'impression de se remplir progressivement à partir de 8 heures du matin pour connaître une forte animation au milieu de la journée. Le repas du midi étant moins important aux États-Unis que chez nous, il peut souvent se réduire à un sandwich et un fruit pour une grande majorité d'étudiants. Un campus dispose de plusieurs cafétérias ayant chacune leur spécificité en fonction de la clientèle à laquelle elle s'adresse, (étudiants gradués ou non gradués) et de la spécialisation de l'offre alimentaire (repas chaud ou froid, cafés, cookies et boissons uniquement). Aussi à UCLA les cafétérias situées au nord sont plus fréquentées par les étudiants gradués, les chercheurs et les professeurs en raison notamment de la proximité de la bibliothèque de recherche. Les enseignants de UCLA ont certes accès à un restaurant qui leur est réservé, mais en fait, ils ne le fréquent qu'occasionnellement, préférant parfois partager le temps du repas avec des étudiants. Il est utile de préciser que de nombreux séminaires informels se déroulent à l'heure de la pause du déjeuner, ce qui oblige étudiants et enseignants à apporter avec eux leur sandwich.

Le campus donne l'impression de commencer à se vider à partir de $18 \mathrm{~h}$ même si les cafétérias accueillent jusqu'à $20 \mathrm{~h}$ des étudiants pour le repas du soir. Il est certain que les étudiants (non gradués) vivant dans les dorms (bâtiments résidentiels incluant des chambres à partager à deux ou trois) fréquentent ces cafétérias ainsi que les chercheurs qui utilisent la bibliothèque en soirée. Les deux grandes bibliothèques, qui ne ferment qu'à $23 \mathrm{~h}$, sont encore bien éclairées et il n'est pas rare de rencontrer des piétons sur le chemin reliant les bibliothèques au parking. Ces deux bibliothèques sont fréquentées non seulement par les étudiants et les professeurs mais par tout individu qui paie des droits d'accès. Notons que des étudiants qui font des stages dans des entreprises ou encore qui y travaillent à mi-temps ont ainsi accès à la 
bibliothèque et aux services de la bibliothèque en soirée. L'accès aux revues scientifiques et à la documentation grâce aux technologies récentes n'a pas véritablement réduit l'animation suscitée par la fréquentation des bibliothèques. Il est vrai que pour de nombreux étudiants, la bibliothèque représente tout autant un cadre pour travailler qu'un lieu où on peut emprunter des ouvrages.

Le campus est certainement moins animé en soirée mais il n'est pas pour autant déserté, en raison des heures d'ouvertures de la bibliothèque et des manifestations culturelles (non gratuites) qui s'y déroulent (concert, récital, conférences). Ces manifestations sont fréquentées en majorité par des habitants de la ville, venues de l'extérieur du campus.

Les professeurs ne vivent pas sur le campus, seuls les étudiants undergraduate dont les parents vivent en dehors de Los Angeles ont accès aux dormitories localisés au nord-ouest du campus en haut de la colline. Ces bâtiments sont certainement les plus élevés du campus. La grande majorité des étudiants vit en dehors du campus dans des appartements qu'ils louent en co-location ou des chambres qu'ils louent chez des particuliers habitant dans une maison entourée d'un jardin ${ }^{2}$. Ce cluster de dormitories est relativement bien animé les vendredis et samedis soirs lorsque les étudiants se détendent et font la fête. L'animation est toutefois bien plus limitée que dans les fraternities et sororities localisées à l'opposé du campus dans le secteur sud-est. Les étudiants vivant dans les fraternities et sororities ont des liens plus étroits entre eux dans la mesure où ils partagent des tâches domestiques, mais ils ont également la réputation de boire de manière démesurée en fin de semaine, ce dont les riverains situés à proximité ne se privent pas de se plaindre. Il est vrai aussi que ces jeunes étudiants qui vivent pour la première fois de leur vie en dehors de chez leurs parents, viennent de différents Etats et font en quelque sorte l'apprentissage et l'expérience de la vie en société (ou de leur autonomie?).

Comme tout campus, UCLA dispose de sa propre police, d'une équipe de pompiers et de vigiles. Un service d'escorte est ainsi proposé à toute personne (notamment les femmes) souhaitant se rendre du bâtiment où il/elle travaille au parking après $22 \mathrm{H}$.

Comment se construit le rapport ou la relation entre le campus et la ville, notamment dans une grande ville comme Los Angeles?

Cynthia Ghorra Gobin : La relation ou encore le rapport entre le campus et la ville est d'une grande complexité. Le campus est a priori ouvert sur la ville et tout habitant de LA peut $\mathrm{y}$ avoir accès librement.

Pour les losangelinos, le campus fait l'objet d'une grande fierté. Le campus UCLA se caractérise par la qualité d'un aménagement doté d'une valeur esthétique partagé de tous et s'avère extrêmement plaisant pour les piétons. Il symbolise la présence de l'Etat californien et la vitalité d'une institution publique participant au progrès social et technologique et de ce fait à l'avenir de l'humanité. Ce sentiment de fierté continue de s'imposer en dépit des changements intervenus dans les financements de l'université : la participation de l'état fédéré californien au budget de l'université n'a cessé de diminuer au profit d'autres financements publics (Etat fédéral notamment) et notamment d'entreprises privées pour la recherche. La hausse des frais de scolarité pour les étudiants californiens et les étudiants non-californiens alimente par ailleurs un sérieux débat sur les conditions d'accès à l'université. 
La ville de Los Angeles inclut plusieurs campus dont les deux plus importants sont USC et UCLA. Les losangelinos sont également fiers de l'ensemble des campus parce qu'ils représentent de hauts lieux culturels. L'orchestre philharmonique de Los Angeles joue régulièrement à UCLA et de nombreux artistes et de musiciens concertistes (de passage à Los Angeles) se produisent également à UCLA. A cette attractivité culturelle s'ajoute celle qui repose sur l'organisation de manifestations sportives.

Comment résumer en guise de conclusion les principales caractéristiques du campus?

Un campus américain est pensé comme une entité autonome: on peut sans trop exagérer évoquer le terme "self-sufficiency », en dehors bien entendu de la fonction résidentielle. Tout est fait pour que l'étudiant, l'enseignant, le chercheur et le personnel administratif trouve sur le campus les produits et les services dont il/elle a besoin. Dans les services sont inclus outre les cafétérias, les librairies et la boutique de cadeaux, l'accès à des activités de détente (gymnase, piscine).

Il est certes un haut lieu pour la recherche et l'enseignement supérieur mais il est également un haut lieu culturel et sportif fréquenté par la bourgeoisie de LA, incluant également les Alumni qui sont souvent des philanthropes soucieux de l'avenir de la recherche.

Pour les Américains, le campus ne se réduit pas uniquement à un «marché aux idées» (a marketplace of ideas), expression souvent utilisée pour souligner la place accordée à la recherche scientifique fondée sur la créativité et l'innovation. Il se doit également d'offrir un cadre mettant en scène à travers son aménagement les valeurs de la société américaine et plus particulièrement son attachement à l'idée de nature, un élément fort de son identité.

\section{BIBLIOGRAPHIE}

Nussbaum M.C., Not for Profit: why democracy needs the humanities, Princeton University press, 2010.

Olmsted F.L., Civilizing American Cities: writing on city landscapes, De Capo, 1997.

\section{NOTES}

1. Les parkings sont tous situés en périphérie du campus, y compris celui des enseignants et du personnel administratif. Ce qui signifie que tout automobiliste fait également un trajet à pied.

2. Ces chambres sont spacieuses, bénéficient d'une salle de bains et peuvent avoir un accès direct sur le jardin. 


\section{AUTEURS}

\section{CAPUCINE BOIDIN}

Maître de conférences en anthropologie à l'Université Sorbonne Nouvelle Paris 3, IHEAL (Institut des Hautes Etudes d'Amérique Latine). Chargée de cours de guarani à l'INALCO (Institut National des Langues et Civilisations Orientales), ses axes de recherche portent sur le métissage, le genre, les mémoires de guerre. Elle a publié en 2010 un numéro des Cahiers des Amériques latines sur le tournant décolonial.

http://www.ladocumentationfrancaise.fr/catalogue/3303332400621/index.shtml ; http:// www.iheal.univ-paris3.fr/spip.php?article1041

\section{CYNTHIA GHORRA-GOBIN}

Directeur de recherche au CNRS. Rattachée au Creda, elle enseigne à l'Iheal (Université de Paris 3 Sorbonne nouvelle). Sa recherche est centrée sur la ville (une construction sociale et matérielle indissociable de la culture et de la dynamique politique) à partir d'un travail de terrain mené sur les villes aux Etats-Unis. Elle travaille actuellement sur la métropolisation dans une perspective comparative (Amérique latine/Amérique du Nord) et dirige le Dictionnaire critique de la mondialisation (Colin, 2012).

implementation(at)orangepointfr 